\title{
Associação entre o escore de Alvarado, achados cirúrgicos e aspecto histopatológico da apendicite aguda.
}

\section{Association between the Alvarado score and surgical and histopathological findings in acute appendicitis.}

Ricardo Reis do Nascimento'; Jaime César Gelosa Souza, TCBC-SC1; Vanessa Baschirotto Alexandre'; Kelser de Souza Kock'; Darlan de Medeiros Kestering ${ }^{1}$

R E S U M O

Objetivo: comparar o resultado do escore de Alvarado com os achados cirúrgicos e com os resultados do exame histopatológico do apêndice de pacientes operados por apendicite aguda. Métodos: estudo observacional com delineamento transversal de 101 pacientes com 14 anos de idade ou mais, submetidos à apendicectomia de urgência. A avaliação continha o escore de Alvarado, pontuação no escore, sexo, idade, etnia dos pacientes e tempo de evolução. Foi obtido o aspecto cirúrgico do apêndice, dados a respeito das complicações pós-operatórias e o resultado do exame histopatológico. O intervalo de confiança pré-estabelecido foi de $95 \%$. Foram calculadas sensibilidade, especificidade, valor preditivo positivo e negativo do escore, e realizada uma análise através da curva ROC. Resultados: a associação entre o escore de Alvarado e a confirmação diagnóstica utilizando como ponto de corte uma pontuação maior ou igual a seis encontrou-se significância estatística $(P=0,002)$, com sensibilidade de $72 \%$ e especificidade de $87,5 \%$. A pontuação maior ou igual a seis mostrou maior tendência a apresentar fases mais avançadas da apendicite aguda tanto no aspecto cirúrgico quanto histopatológico, quando comparado a uma pontuação menor que seis. O sexo masculino apresentou maiores chances de complicações quando comparado ao sexo feminino $(P=0,003)$. Conclusão: o escore de Alvarado se mostrou um bom método para triagem diagnóstica na apendicite aguda, já que pontuações maiores ou iguais a seis estão associadas a uma probabilidade maior de confirmação diagnóstica e de quadros mais avançados da doença aguda.

Descritores: Apendicite. Apendicectomia. Diagnóstico. Emergências.

\section{INTRODUÇÃO}

dor abdominal é a apresentação mais prevalente
no atendimento emergencial ${ }^{1}$, sendo a apendicite aguda (AA) a causa mais comum de urgência abdominal ${ }^{2}$ e a apendicectomia, padrão ouro de tratamento da $A \mathrm{~A}$, a cirurgia de emergência mais realizada no mundo ${ }^{3}$. Cerca de 90 a 100 pacientes por 100.000 habitantes terão esta doença por ano ${ }^{4} \mathrm{e}$ estima-se que o risco de desenvolver AA ao longo da vida esteja entre $7 \%$ e $8 \% 3$, sendo $8,6 \%$ em homens e $6,7 \%$ em mulheres ${ }^{5}$. Essa incidência é maior em adolescentes e adultos jovens, estando a população mais afetada entre 25 e 35 anos de idade ${ }^{6}$.

A forma clássica de AA pode ser prontamente diagnosticada e tratada. No entanto, a presença de características atípicas pode dificultar o diagnóstico, uma vez que os sintomas típicos e as alterações laboratoriais compatíveis podem estar ausentes em $20 \%$ a $33 \%$ dos pacientes, principalmente durante os estágios iniciais ${ }^{1}$.
Em tais casos, a investigação de imagem pode ser útil para estabelecer um diagnóstico correto ${ }^{7}$. Dentre os exames, há um papel limitado no exame radiológico, sendo este útil para descartar outras doenças que causem abdome agudo. A ultrassonografia possui uma alta taxa de resultados falsos positivos e negativos ${ }^{8}$. A tomografia computadorizada é o exame de escolha por possuir alta sensibilidade e especificidade para o diagnóstico ${ }^{9}$, porém tem alto custo e não está disponível em todos os centros de atendimento. Além disso, em casos de AA típica, seu uso pode atrasar a apendicectomia e aumentar o risco de perfuração ${ }^{10}$. $\mathrm{O}$ método definitivo para confirmação do diagnóstico de AA é o exame histopatológico do apêndice ${ }^{11}$.

O diagnóstico clínico pode levar a uma taxa de apendicectomia não terapêutica de $15 \%$ a $30 \%{ }^{12}$ e a taxa de AA perfurada não diagnosticada pode chegar a 3,4\%, uma vez que os sintomas de AA podem se sobrepor a sintomas urológicos, abdominais e ginecológicos ${ }^{13}$. Assim, o diagnóstico tardio ou

1 - UNISUL - Universidade do Sul de Santa Catarina, Faculdade de Medicina, Tubarão, SC, Brasil. 
incorreto pode resultar em múltiplas complicações, como infecções do sítio cirúrgico, perfuração, abcessos, sepse e morte ${ }^{14}$. O diagnóstico correto e a intervenção cirúrgica de forma precoce são os melhores métodos para reduzir morbimortalidade, o tempo de hospitalização e os custos do tratamento ${ }^{15}$.

É relevante incorporar na prática clínica testes como escores que auxiliam no diagnóstico de $A A^{16}$. Existem inúmeras classificações de risco cujo objetivo é identificar pacientes de baixo, médio e alto risco para AA, permitindo que as investigações posteriores sejam estratificadas de acordo com o mesmo ${ }^{17}$. Dentre tais testes, o escore de Alvarado foi elaborado com a intenção de diminuir o número de exames de imagem solicitados ${ }^{18}$. Alvarado descreveu um sistema de pontuação com base em oito fatores clínicos preditivos para melhorar a avaliação no diagnóstico de AA, que produz uma pontuação total máxima de dez pontos e inclui sintomas e sinais clínicos, e achados laboratoriais ${ }^{19}$.

O presente estudo tem como objetivo comparar o resultado do escore de Alvarado com os achados cirúrgicos e com os resultados do exame histopatológico de pacientes operados por apendicite aguda.

\section{MÉTODOS}

O estudo segue as diretrizes e normas regulamentadoras de pesquisa envolvendo seres humanos propostas pela Resolução № 466/2012, do Conselho Nacional de Saúde. Os dados foram coletados após a aprovação do Comitê de Ética em Pesquisa da Unisul - CEP sob o parecer 2.362.539, e mediante assinatura do termo de consentimento pelos participantes e por seus responsáveis em casos de menores de 18 anos de idade. Trata-se de um estudo observacional com delineamento transversal. A amostra foi composta por 101 pacientes a partir de 14 anos de idade, com quadro suspeito de apendicite aguda e submetidos à apendicectomia no período compreendido entre primeiro de abril e 30 de setembro de 2017, atendidos em um hospital do sul de Santa Catarina. Foram excluídos aqueles em que faltavam dados e aqueles incapacitados de fornecerem as informações necessárias ao estudo.

A entrevista aplicada continha o sexo, a idade e a etnia dos pacientes, o tempo de evolução e o escore de Alvarado, que inclui migração da dor, anorexia, náuseas e/ou vômitos, dor à descompressão em fossa ilíaca direita (FID), aumento da temperatura e desvio à esquerda ao leucograma, somando um ponto a cada critério preenchido, leucocitose e defesa em quadrante abdominal inferior direito somando dois pontos cada ${ }^{19}$. Após o término do período de coleta utilizando as entrevistas, foi consultado o prontuário eletrônico a fim de obter o aspecto cirúrgico do apêndice e os dados a respeito das possíveis complicações pós-operatórias. Posteriormente, foi acessado o sistema de dados do laboratório de referência da região para consultar o resultado do exame histopatológico, que fornecia o laudo com a classificação em apêndice normal, AA incipiente, $A A, A A$ purulenta e $A A$ gangrenosa ${ }^{20}$. A pontuação do escore de Alvarado foi calculada no momento de construção do banco de dados.

Os dados foram arquivados e tabulados em planilha eletrônica, utilizando o programa Epilnfo 3.5.4 e analisados através do programa estatístico SPSS (Statistical Package for the Social Sciences) versão 20.0. Os dados quantitativos foram apresentados em medidas de tendência central e dispersão e os qualitativos em percentuais e em números absolutos. Para verificar a associação entre as variáveis de interesse, foi aplicado o teste Qui-quadrado para a comparação de proporções e teste $T$ de Student ou teste de Man-Whitney para comparação das médias. O intervalo de confiança pré-estabelecido foi de $95 \%, p=0,05$. Foram calculados sensibilidade e especificidade do escore na população estudada, bem como, seu valor preditivo positivo e negativo, e, então, realizada uma análise através da curva ROC. 


\section{RESULTADOS}

Dos 101 pacientes avaliados, 49 pacientes eram do sexo feminino $(48,5 \%)$ e 52 do sexo masculino (51,5\%). A mediana de idade foi de 29 anos, com distância interquartílica de 19. Quanto à etnia, a maior parte dos pacientes era de caucasiano, sendo estes $92,1 \%$.

Com relação ao escore de Alvarado, a apresentação mais frequente foi dor à descompressão súbita em fossa ilíaca direita em 92\%, seguido de leucocitose em $84,2 \%$, anorexia em $77,2 \%$, náuseas e/ou vômitos em 75,2\%. A migração da dor, defesa em quadrante inferior direito do abdome, elevação da temperatura, e desvio à esquerda ao leucograma estiveram presentes em menor frequência, sendo estas, respectivamente, $56,4 \%$, 47,5\%, 38,6\% e $15,8 \%$.

Para realização da avaliação do escore de Alvarado, cálculo da sensibilidade e especificidade, valor preditivo positivo (VPP) e valor preditivo negativo (VPN), bem como, para a associação do escore com as demais variáveis do estudo, foram adotados como pontos de corte um escore maior ou igual a cinco, seis e sete, de acordo com a curva ROC, que indicou um ponto de corte maior que 5,5 como sendo significativo estatisticamente (Figura 1). Dessa forma, 86,1\% obteve uma pontuação maior ou igual a cinco, $67,3 \%$, uma pontuação maior ou igual a seis e $36,6 \%$, maior ou igual a sete.

Considerando a análise dos dados do escore de Alvarado dos 101 participantes observou-se que: $2 \%$ fez dois pontos, $1 \%$, três pontos, 10,9\%, quatro pontos, $18,8 \%$, cinco pontos, 30,7\%, seis pontos, $17,8 \%$, sete pontos, $5,9 \%$, oito pontos, $10,9 \%$, nove pontos e $2,0 \%$, dez pontos. O escore maior ou igual a seis apresentou sensibilidade e especificidade de $72 \%$ e $87,5 \%$, respectivamente, com VPP de $98,53 \%$ e VPN de $21,21 \%$, com acurácia de 73,27\%. Para o escore maior ou igual a cinco foi encontrado sensibilidade de $88,17 \%$, especificidade de $37,5 \%$, VPP e VPN de $94,25 \%$ e $21,43 \%$, respectivamente.
Para valores maiores ou iguais a sete a sensibilidade, a especificidade, o VPP e o VPN foram, respectivamente, de $38,71 \%, 87,5 \%, 97,3 \%$ e $10,94 \%$.

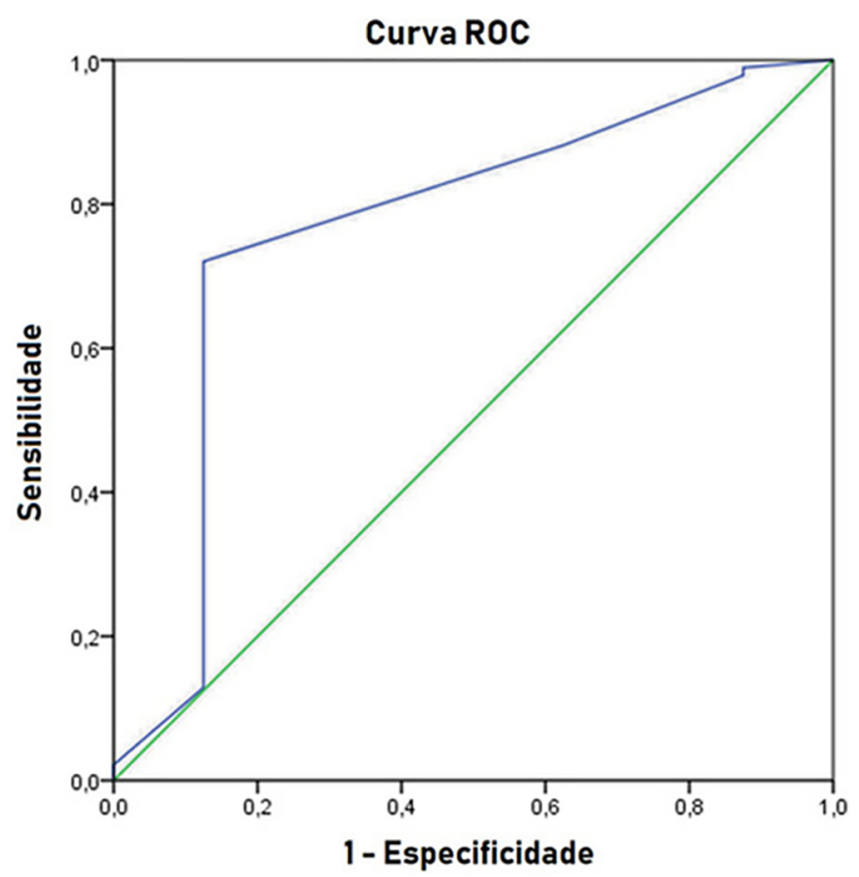

Figura 1. Curva ROC.

O tempo de evolução entre o início da dor e a realização da apendicectomia foi majoritariamente $(77,2 \%)$ entre um e três dias, sendo menor do que um dia para $13,9 \%$ e maior do que três dias para $8,9 \%$. 0 aspecto cirúrgico encontrado foi em sua maior parte nas fases I e II. Com a distribuição das fases em: $4 \%$ fase 0 , $36,6 \%$ fase I, 35,6\% fase II, 20,8\% fase III e 3\% fase IV.

A confirmação diagnóstica com histopatológico foi feita em 92,1\%, sendo a taxa de apendicectomia não terapêutica de 7,9\%, totalizando oito pacientes, dos quais $75 \%$ era do sexo feminino. Os resultados dos achados histopatológicos foram: AA incipiente 7,9\%, AA $53,5 \%$, AA supurativa $29,7 \%$ e AA gangrenosa $1 \%$.

A taxa de complicações pós-operatórias foi de $17,9 \%$, sendo a principal infecção de ferida operatória, seguida de íleo metabólico e febre com, respectivamente, 6,9\%, 5\% e 4\%. Deiscência e hematoma de ferida operatória ocorreram na frequência de $1 \%$ cada. 
Com relação à associação entre o escore de Alvarado e a confirmação diagnóstica, utilizando como ponto de corte uma pontuação maior ou igual a seis, encontrou-se significância estatística $(p=0,002)$, mostrando uma maior chance de diagnóstico de AA para tais resultados. Para os outros pontos de corte adotados não foram encontradas diferenças estatisticamente significantes (Tabela 1).

Associando-se o escore de Alvarado com a idade e sexo e utilizando o ponto de corte maior ou igual a seis, não se encontrou associação com significância estatística (Tabela 2).
Não se encontrou significância estatística na associação entre a pontuação no escore de Alvarado, os aspectos cirúrgicos e histopatológicos da AA (Tabela 3). Entretanto, percebeu-se que a pontuação do escore maior ou igual a seis mostrou maior tendência a apresentar fases mais avançadas da AA, tanto no aspecto cirúrgico quanto histopatológico, comparado a um escore menor do que seis.

Com relação ao aspecto cirúrgico e histopatológico, percebeu-se que houve discordância entre os achados, principalmente entre as fases 0,1 e II, com o aspecto histopatológico. O aspecto cirúrgico

Tabela 1. Associação entre o escore de Alvarado e a confirmação diagnóstica pelo histopatológico.

\begin{tabular}{cccc}
\hline Pontuação no escore & Confirmação diagnóstica & Ausência de confirmação & $p$ \\
\hline$<5$ & $11(11,8 \%)$ & $3(37,5 \%)$ & \\
$\geq 5$ & $82(88,2 \%)$ & $5(62,5 \%)$ & 0,079 \\
$<6$ & $26(92,8 \%)$ & $7(87,5 \%)$ & \\
$\geq 6$ & $67(72 \%)$ & $1(12,5 \%)$ & 0,002 \\
$<7$ & $57(61,3 \%)$ & $1(12,5 \%)$ & \\
$\geq 7$ & $36(38,7 \%)$ & $1(12,5 \%)$ & 0,252 \\
\hline
\end{tabular}

Tabela 2. Associação do escore de Alvarado com os dados sociodemográficos.

\begin{tabular}{lccc}
\hline \multicolumn{1}{c}{ Variável } & Pontuação $<6$ & Pontuação $\geq 6$ & $p$ \\
\hline Sexo & & & \\
Masculino & $20(40,8 \%)$ & $29(59,2 \%)$ & 0,090 \\
Feminino & $33(25 \%)$ & $23(88,5 \%)$ & \\
Idade & $26 \pm 20$ & $29 \pm 18$ & 0,789 \\
\hline
\end{tabular}

Tabela 3. Associação entre o escore de Alvarado, aspecto cirúrgico e histopatológico.

\begin{tabular}{lcc}
\hline Variável & Pontuação <6 & Pontuação $\geq 6$ \\
\hline Aspecto Cirúrgico & $3(75 \%)$ & $1(25 \%)$ \\
Fase 0 & $11(29,7 \%)$ & $26(70,3 \%)$ \\
Fase I & $13(36,1 \%)$ & $23(63,9 \%)$ \\
Fase II & $6(28,6 \%)$ & $15(71,4 \%)$ \\
Fase III & $0(0 \%)$ & $3(100 \%)$ \\
Fase IV & & \\
Histopatológico & $2(25 \%)$ & $6(75 \%)$ \\
AA incipiente & $15(27,8 \%)$ & $39(72,2 \%)$ \\
AA & $9(30 \%)$ & $21(70 \%)$ \\
AA supurativa & $0(0 \%)$ & $1(100 \%)$ \\
AA gangrenosa & & \\
\hline
\end{tabular}


relacionado com a confirmação diagnóstica pelo histopatológico pode ser encontrado na tabela 4.

Não foi observado relação entre a pontuação no escore de Alvarado e a presença de complicações com nenhum dos pontos de corte adotados. Porém, mesmo que de forma não estaticamente significativa, percebeu-se que usando os pontos de corte maior ou igual a cinco e seis, os casos que complicaram neste estudo estavam presentes quanto maior era o escore (Tabela 5).

Dos pacientes que apresentaram complicações, a maior parte apresentava AA supurativa ao histopatológico, sendo estes 55,5\%, seguidos de AA com 16,6\%, AA incipiente e apêndice normal com $11,1 \%$ cada e AA gangrenosa com 5,5\%. Nestes mesmos pacientes a fase cirúrgica mais encontrada foi a fase III, com 38,8\%, seguido da fase I, com 33,3\%, fase II, com 22,2\%, e fases 0 e IV, com 5,5\% cada.

Não houve significância estatística na associação do tempo de evolução com nenhuma das variáveis, nem mesmo com a presença de complicações. Dos pacientes que apresentaram complicações, 5,5\% teve evolução menor do que um dia, 16,6\%, evolução prolongada, maior do que três dias e $77,7 \%$, evolução de um a três dias.
Dos que tiveram quatro ou mais dias de evolução, 37,5\% apresentou complicações.

Não houve associação das complicações pósoperatórias com nenhuma outra variável além do sexo, em que o masculino apresentou maiores chances de complicações quando comparado ao sexo feminino ( $P=0,003)$, representando 83,3\% das complicações.

\section{DISCUSSÃO}

No presente estudo, os casos de AA foram mais prevalentes no sexo masculino, estando em consonância com estudos semelhantes ${ }^{21-24}$, bem como, a mediana de idade encontrada, que foi de 29 anos, semelhante a estudos onde a prevalência de AA se dá na segunda e terceira décadas ${ }^{7,21,23,24}$.

A frequência de cada um dos critérios do escore de Alvarado foi semelhante aos achados de Memon et al. ${ }^{25}$ e Rodrigues e Sindhu²6, mas diferiu dos de Swami et al. ${ }^{24}$ que observaram predominância de defesa em FID e da migração da dor, e menor presença de leucocitose. Assim como no estudo brasileiro de Sousa-Rodrigues et al. ${ }^{21}$, em que a elevação da temperatura ocorreu numa proporção maior, 85,1\% contra 38,6\%, diferindo também na dor à descompressão, e na presença de

Tabela 4. Aspecto cirúrgico e confirmação diagnóstica.

\begin{tabular}{|c|c|c|}
\hline Aspecto cirúrgico & Confirmação diagnóstica & Ausência de confirmação \\
\hline Fase 0 & $0(0 \%)$ & $4(100 \%)$ \\
\hline Fase I & $33(89,2 \%)$ & $4(10,8 \%)$ \\
\hline Fase II & $36(100 \%)$ & $0(0 \%)$ \\
\hline Fase III & $21(100 \%)$ & $0(0 \%)$ \\
\hline Fase IV & $3(100 \%)$ & $0(0 \%)$ \\
\hline
\end{tabular}

Tabela 5. Associação entre o escore de Alvarado e as complicações pós-operatórias.

\begin{tabular}{cccc}
\hline Pontuação no escore & Presença de complicações & Ausência de complicações & $p$ \\
\hline$<5$ & $1(5,6 \%)$ & $70(84,3 \%)$ & \\
$\geq 5$ & $17(94,4 \%)$ & $13(15,7 \%)$ & 0,454 \\
$<6$ & $6(33,3 \%)$ & $27(32,5 \%)$ & 1 \\
$\geq 6$ & $12(66,7 \%)$ & $56(67,5 \%)$ & \\
$<7$ & $12(66,7 \%)$ & $52(62,7 \%)$ & 0,795 \\
$\geq 7$ & $6(33,3 \%)$ & $31(37,3 \%)$ & \\
\hline
\end{tabular}


defesa em quadrante inferior direito. Já quanto à leucocitose, encontrou-se resultados semelhantes. As divergências observadas podem estar relacionadas com a diferença entre as populações estudadas e a forma de avaliação dos critérios.

De acordo com a distribuição da pontuação do escore de Alvarado, as mais prevalentes foram seis, cinco e sete pontos, sendo que a maior parte da população estudada apresentou uma pontuação maior ou igual a cinco, pontuações mais baixas do que as encontradas no estudo de SousaRodrigues et al. ${ }^{21}$, em que as mais prevalentes foram, respectivamente, sete, oito e seis pontos. Abdelrahim et al. ${ }^{27}$ apresentaram uma prevalência da pontuação maior ou igual a sete, e o mesmo aconteceu ao comparar-se os dados com os de um estudo da população sul africana ${ }^{28}$, que somou pontuações maiores no escore. Já Memon et al. ${ }^{25}$, encontraram dados semelhantes, em que a maioria dos pacientes obteve um escore de cinco ou seis.

Jalil et al. ${ }^{7}$ e Cedillo-Alemán et al. ${ }^{18}$ tiveram achados coincidentes com o presente estudo quanto à sensibilidade, especificidade, VPP e VPN. Memon et al. ${ }^{25}$ encontraram VPP e VPN maiores. O estudo de Genzor Ríos et al. ${ }^{22}$ comparou sensibilidade e VPP para valores do escore maiores ou iguais a cinco e a sete, sendo aqueles maiores quando o ponto de corte foi menor. No presente estudo observouse que quando se aumenta o ponto de corte, a sensibilidade e o VPN caem, mas a especificidade e VPP aumentam. O inverso é observado quando se usa valores menores como ponto de corte.

Houve associação entre o escore de Alvarado e a confirmação diagnóstica pelo histopatológico utilizando o ponto de corte maior ou igual a seis, sendo que a taxa de apendicectomia não terapêutica foi de 7,9\%, predominantemente no sexo feminino. Comparando esses achados com a literatura observou-se semelhança com os achados de Swami et al. ${ }^{24}$, em que um escore maior que sete resultou em confirmação diagnóstica em
90,9\%, e com Lima et al. ${ }^{23}$, em que $98,75 \%$ dos apendicectomizados confirmaram AA e dos que não confirmaram, $75 \%$ era do sexo feminino, o que pode ser explicado devido à sobreposição de sintomas ginecológicos no sexo feminino ${ }^{13}$. Genzor Ríos et al.22 obtiveram uma taxa de apendicectomia não terapêutica semelhante, de 5,68\%, porém sem distinção entre os sexos. Ao contrário, Jalil et al. ${ }^{7}$ observaram uma taxa de apendicectomia não terapêutica maior em homens.

Em estudo de Quesada Suárez et al. ${ }^{29} \mathrm{O}$ diagnóstico foi confirmado em $86 \%$ e, daqueles submetidos à apendicectomia não terapêutica, a maioria apresentava pontuações entre cinco e sete, assim como em trabalho de Abdelrahim et al. ${ }^{27}$, em que todos os pacientes submetidos à apendicectomia não terapêutica tiveram escore abaixo de sete. No presente estudo, todos os pacientes não confirmados tinham um escore menor ou igual a cinco, com exceção de um paciente que somou nove pontos.

Estudos com maiores discordâncias encontrados foram o de Rodrigues e Sindhu ${ }^{26}$, no qual a maioria dos pacientes com diagnóstico confirmado possuía um escore de menos que 7,8, contrariando os demais estudos que associam pontuações mais altas à confirmação diagnóstica, e Memon et al. ${ }^{25}$, cuja taxa apendicectomia não terapêutica foi de $28,7 \%$, números relativamente maiores do que os do presente estudo. Tal discrepante divergência pode ser atribuída à diferença entre as populações estudadas e à amostra pré-selecionada contida neste estudo, onde os pacientes incluídos foram aqueles submetidos à apendicectomia.

O estudo de Jalil et al. ${ }^{7}$ mostrou que pacientes com escore maior ou igual a sete apresentaram maiores chances de possuírem fases mais avançadas da AA no histopatológico. No presente estudo, mesmo sem associação estatística, a pontuação do escore maior ou igual a seis mostrou maior tendência a apresentar fases mais avançadas de AA no histopatológico, 
comparado a um escore menor do que seis, sendo que tais observações também foram feitas por Ospina et al. ${ }^{30}$. Os achados histopatológicos deste estudo foram principalmente AA e AA supurativa, estando em consonância com Sudhir et al..31.

O aspecto cirúrgico encontrado foi, em sua maior parte, nas fases I e II. Para Swami et al. ${ }^{24}$, a maioria dos apêndices apresentavam apenas características inflamatórias, representando estágios iniciais da AA, concordando com o presente estudo, bem como, os de Silva et al. ${ }^{32}$ e Nutels et al. ${ }^{33}$, estudos brasileiros a respeito das complicações da $A A$, que encontraram fases predominantemente iniciais. No estudo de caso controle nacional de lamarino et al. ${ }^{34}$ observou-se maior predominância das fases flegmonosa e gangrenosa e, em SousaRodrigues et al. ${ }^{21}$, as fases mais prevalentes foram III e II, respectivamente, consideradas ligeiramente mais avançadas do que as por nós encontradas. Sousa-Rodrigues et al. ${ }^{21}$ mostraram correlação entre o escore de Alvarado e o aspecto cirúrgico de forma estatisticamente significativa, diferente dos achados do presente estudo, no qual não se encontrou relevância estatística em tal associação. Percebeuse, no entanto, que utilizando o ponto de corte maior ou igual a seis, este mostrou maior tendência a apresentar fases mais avançadas da AA no aspecto cirúrgico quando comparado a um escore menor do que seis. Discordando de forma significante, o estudo da população sul africana, de Kong et al.28, obteve predominantemente fases avançadas, já com perfuração. Tal discrepância pode estar relacionada com as diferenças encontradas entre as populações de ambos os estudos, bem como, a disponibilidade de acesso a serviços de saúde.

No estudo de Sousa-Rodrigues et al..$^{21}$ o tempo entre início dos sintomas e a apendicectomia foi de aproximadamente $32,4 \pm 5,4$ horas, dados semelhantes aos encontrados neste estudo. Ospinal et al..$^{30}$ mostraram relação entre o tempo de evolução maior do que 36 horas e a presença de necrose, diferente do presente estudo, no qual não houve associação do tempo de evolução com o diagnóstico ou com a presença de necrose. Em Genzor Ríos et al. ${ }^{22}$ o tempo de evolução também não apresentou relação com diagnóstico. Achados em Nutels et al.33 mostram que um maior tempo de evolução resultou em maiores complicações, sendo que pacientes com mais de quatro dias de evolução obtiveram índice de complicações de $57,2 \%$. No presente estudo, entre os pacientes que apresentaram complicações, 77,7\% teve evolução entre um e três dias, o que pode ser atribuído à predominância observada desse tempo de evolução. Entre aqueles que tiveram quatro ou mais dias de evolução, 37,5\% apresentou complicações.

Ao se confrontar os achados das complicações com a literatura, observa-se que os mesmos sofrem alterações de acordo com a população estudada e as variáveis que as cercam, como pôde ser observado no estudo sul africano ${ }^{28}$. Neste estudo, a população possuía menores condições socioeconômicas, maior tempo de evolução de doença, 59,5\% tinha $A A$ perfurada e desses, $29,7 \%$ possuía AA perfurada associada à sepse intra-abdominal e 70,2\% tinha associação com sepse generalizada. Ao contrário, em nosso estudo, a taxa de complicações pós-operatórias foi de $17,9 \%$, sendo a principal complicação a infecção de ferida operatória, resultados semelhante aos de Nutels et al. ${ }^{33}$, com taxas de 17,2\% de complicações, sendo, também, a infecção de ferida operatória a mais frequente. Nele, todavia, o desfecho com óbito foi observado em 0,9\%, diferindo do presente estudo, em que a taxa de mortalidade foi zero. Em estudo de Lima et al. ${ }^{23}$ apenas 5,96\% dos pacientes apresentou complicações, sendo a infecção de ferida operatória também a mais frequente. No estudo de caso controle de lamarino et al. ${ }^{34}$, dos pacientes que apresentaram complicações, a mais encontrada foi o abscesso intra-abdominal, seguida de infecções de ferida operatória, diferindo do presente estudo, porém ainda apresentando infecção de ferida operatória como uma complicação significativa. 
No presente estudo, dos pacientes que apresentaram complicações, a maior parte apresentava AA supurativa ao histopatológico e, nestes mesmos pacientes, a fase cirúrgica mais encontrada foi a fase III, concordante com trabalho de Silva et al. ${ }^{32}$, em que pacientes com apêndice necrosado sem perfuração (Fase III), tiveram 3,32 mais chances de terem complicações pósoperatórias. Nutels et al. ${ }^{33}$ demonstraram percentual de complicações pós-operatórias maior também nas fases III e IV e lamarino et al. ${ }^{34}$ observaram que as complicações ocorreram com maior frequência nas fases perfurada e gangrenosa, respectivamente, fases evolutivas ligeiramente mais avançadas do que as encontradas no nosso estudo.

Não foi encontrada relação com significância entre a pontuação no escore de Alvarado e a presença de complicações, porém percebeu-se a existência de valores maiores do escore nos casos que complicaram, associação também vista por Jalil et al.7. No presente estudo, o sexo masculino apresentou maiores índices de complicações, assim como observado por Nutels et al. ${ }^{33}$ e lamarino et al. ${ }^{34}$, em que as complicações foram mais frequentes no sexo masculino, e por Lima et al. ${ }^{23}$, em que a evolução para o óbito foi mais frequente no sexo masculino. Já para Silva et al. ${ }^{32}$, de forma discordante, pacientes do sexo feminino tiveram 1,94 vezes mais chances de terem complicações pós-operatórias.

Diante do exposto, pode se observar que apesar das divergências, o escore de Alvarado representa um bom método para triagem diagnóstica na $A A$, já que pontuações maiores ou iguais a seis estão associadas a uma probabilidade maior de confirmação diagnóstica ao histopatológico. Enfatiza-se ainda, que o escore de Alvarado é um método simples, acessível e de fácil aplicação, que pode acelerar o diagnóstico e, com isto, reduzir o tempo de evolução da doença e das complicações pós-operatórias. Como visto no presente estudo pontuações mais altas podem estar associadas a fases mais avançadas da $A A$, inclusive correlacionando-se com achados mais avançados no exame histopatológico, e a maiores complicações pós-operatórias, demonstrando sua importância.

\title{
A B S T R A C T
}

\begin{abstract}
Objective: to compare the results of the Alvarado score with the surgical findings and the results of the histopathological examination of the appendix of patients operated on for acute appendicitis. Methods: we conducted an observational study with a cross-sectional design of 101 patients aged 14 years and over undergoing emergency appendectomy. The evaluation comprised the Alvarado score, gender, age, ethnicity and time of evolution. We obtained data regarding the surgical aspect of the appendix, postoperative complications and the result of the histopathological examination. The preestablished confidence interval was 95\%. We calculated sensitivity, specificity, positive and negative predictive values of the score, and performed an analysis through the ROC curve. Results: we found a statistically significant ( $p=0.002)$ association between the Alvarado score and the diagnostic confirmation using a cutoff score of six or greater, with a sensitivity of $72 \%$ and a specificity of $87.5 \%$. A score greater than or equal to six showed a greater tendency to present more advanced stages of acute appendicitis in both surgical and histopathological findings when compared with a score lower than six. Males presented greater chances of complications when compared with females $(p=0.003)$. Conclusion: the Alvarado score proved to be a good method for diagnostic screening in acute appendicitis, since scores greater than or equal to six are associated with a higher probability of diagnostic confirmation and more advanced stages of the acute disease.
\end{abstract}

Keywords: Appendectomy. Diagnosis. Emergencies. Appendicitis.

\section{REFERÊNCIAS}

1. Kirkil C, Karabulut K, Aygen E, Ilhan YS, Yur M, Binnetoglu K, et al. Appendicitis scores may be useful in reducing the costs of treatment for right lower quadrant pain. Ulus Travma Acil Cerrahi Derg. 2013;19(1):13-9.
2. Quevedo Guanche L. Apendicitis aguda: clasificación, diagnóstico y tratamiento. Rev Cubana Cir. 2007;46(2). Disponible en: http://scielo.sld. cu/scielo.php?script=sci_arttext\&pid=S0034$74932007000200011 \& \operatorname{lng}=\mathrm{es}$

3. Stewart B, Khanduri P, McCord C, Ohene-Yeboah $M$, Uranues $S$, Vega Rivera $F$, et al. Global disease 
burden of conditions requiring emergency surgery. Br J Surg. 2014;101(1):e9-22.

4. Bhangu A, Søreide K, Di Saverio S, Assarsson JH, Drake FT. Acute appendicitis: modern understanding of pathogenesis, diagnosis, and management. Lancet. 2015;386(10000):1278-87.

5. Humes DJ, Simpson J. Clinical Presentation of Acute Appendicitis: Clinical Signs-Laboratory FindingsClinical Scores, Alvarado Score and Derivate Scores. In: Keyzer C, Gevenois, editors. Medical Radiology. Imaging of Acute Appendicitis in Adults and Children. Berlin, Heidelberg: Springe; 2011. p.13-21.

6. Ilves I, Paajanen $\mathrm{HE}$, Herzig $\mathrm{KH}$, Fagerström A, Miettinen PJ. Changing incidence of acute appendicitis and nonspecific abdominal pain between 1987 and 2007 in Finland. World J Surg. 2011;35(4):731-8.

7. Jalil A, Shah SA, Saaiq M, Zubair M, Riaz U, Habib Y. Alvarado scoring system in prediction of acute appendicitis. J Coll Physicians Surg Pak. 2011;21(12):753-5.

8. Al-Ajerami Y. Sensitivity and specificity of ultrasound in the diagnosis of acute appendicitis. East Mediterr Health J. 2012;18(1):66-9.

9. Krajewski S, Brown J, Phang PT, Raval M, Brown CJ. Impact of computed tomography of the abdomen on clinical outcomes in patients with acute right lower quadrant pain: a meta-analysis. Can J Surg. 2011;54(1):43-53.

10. Busch M, Gutzwiller FS, Aellig S, Kuettel R, Metzger $U$, Zingg $U$. In-hospital delay increases the risk of perforation in adults with appendicitis. World J Surg. 2011.35(7):1626-33.

11. Soomro AG, Siddiqui FG, Abro AH, Abro S, Shaikh NA, Memon AS. Diagnostic accuracy of Alvarado Scoring System in acute appendicitis. J Liaquat Univ Med Heal Sci. 2008;7(2):93-6.

12. Seetahal SA, Bolorunduro OB, Sookdeo TC, Oyetunji TA, Greene WR, Frederick W, et al. Negative appendectomy: a 10-year review of a nationally representative sample. Am J Surg. 2011;201(4):433-7.

13. Park JS, Jeong JH, Lee JI, Lee JH, Park JK, Moon HJ. Accuracies of diagnostic methods for acute appendicitis. Am Surg. 2013;79(1):101-6.
14. Thuijls G, Derikx JP, Prakken FJ, Huisman B, van Bijnen Ing AA, van Heurn EL, et al. A pilot study on potential new plasma markers for diagnosis of acute appendicitis. Am J Emerg Med. 2011;29(3):256-60.

15. Limpawattanasiri $C$. Alvarado score for the acute appendicitis in a provincial hospital. J Med Assoc Thail. 2011;94(4):441-9.

16. Reddy GB, Subramanyam VV, Veersalingam B, Sateesh S, Bangla G, Rao PS. Role of Alvarado score in the diagnosis of acute appendicitis. Int J Res Med Sci. 2013;1(4):404-8.

17. Leeuwenburgh MM, Stockmann HB, Bouma WH, Houdijk AP, Verhagen MF, Vrouenraets $B$, et al. A simple clinical decision rule to rule out appendicitis in patients with nondiagnostic ultrasound results. Acad Emerg Med. 2014;21(5):488-96.

18. Cedillo-Alemán EJ, Santana-Vela IA, GonzálezCano R, Onofre-Castillo J, Gartz-Tondorf GR. Sensibilidad y especificidad de la escala de Alvarado en el diagnóstico de apendicitis aguda comparada con TAC o ultrasonido en las primeras 24 horas de evolución. Cir Gen. 2012;34(2):169-73.

19. Alvarado A. A practical score for the early diagnosis of acute appendicitis. Ann Emerg Med. 1986;15(5):557-64.

20. Mills SE, editor. Sternberg's diagnostic surgical pathology. 5th ed. Baltimore; Philadelphia: Wolters Kluwer; Lippincott Williams \& Wilkins; 2010.

21. Sousa-Rodrigues $C F$, Rocha $A C$, Rodrigues $A K B$, Barbosa FT, Ramos FWS, Valões SHC. Correlation between the Alvarado Scale and the macroscopic aspect of the appendix in patients with appendicitis. Rev Col Bras Cir. 2014;41(5):336-9.

22. Genzor Ríos SJ, Rodríguez Artigas JM, Giménez Maurel T, Vallejo Bernad C, Aguirre Prat N, Miguelena Bobadilla JM. Ecografía y Escala de Alvarado en el diagnóstico de la apendicitis aguda. Impacto en la tasa de apendicectomía negativa. Emergencias. 2016;28(60:396-9.

23. Lima AP, Vieira FJ, Oliveira GP, Ramos PS, Avelino ME, Prado FG, et al. Clinical-epidemiological profile of acute appendicitis: retrospective analysis of 638 cases. Rev Col Bras Cir. 2016;43(4):248-53.

24. Swami G, Kasale R, Khan N. Use of modified Alvarado score and USG combination in decreasing negative appendicectomy rate. IJRTSAT. 2017;22(2):71-4. 
25. Memon ZA, Irfan S, Fatima K, Iqbal MS, Sami W. Acute appendicitis: diagnostic accuracy of Alvarado scoring system. Asian J Surg. 2013;36(4):144-9.

26. Rodrigues $W$, Sindhu S. Diagnostic importance of Alvarado and RIPASA Score in acute appendicitis. Int J Sci Study. 2017;4(11):57-60.

27. Abdelrahim $M$, Khair R, Elsiddig K. The validity of Alvarado Score in diagnosis of acute appendicitis among Sudanese patients. Surgery Curr Res. 2016;6(1):257.

28. Kong VY, van der Linde S, Aldous C, Handley JJ, Clarke DL. The accuracy of the Alvarado score in predicting acute appendicitis in the black South African population needs to be validated. Can J Surg. 2014;57(4):E121-5.

29. Quesada Suárez L, Ival Pelayo M, González Meriño CL. La escala de Alvarado como recurso clínico para el diagnóstico de la apendicitis aguda. Rev Cuba Cir. 2015;54(2):121-8.

30. Ospina J, Barrera L, Manrique F. Utilidad de una escala diagnóstica en casos de apendicitis aguda. Rev Colomb Cir. 2011;26(4):234-41.

31. Sudhir S, Sekhar AP. Evaluation of appendicitis inflammatory response score as a novel diagnostic tool for diagnosis of acute appendicitis and its comparison with Alvarado score. IJSS J Surg. 2017;3(1):21-6.

32. Silva SM, Almeida SB, Lima OAT, Guimarães GMN, Silva ACC, Soares AF. Fatores de Risco para as complicações após apendicectomias em adultos. Rev Bras Coloproct. 2007;27(1):31-6

33. Nutels DBA, Andrade ACG, Rocha AC. Perfil das complicações após apendicectomia em um hospital de emergência. ABCD Arq Bras Cir Dig. 2007;20(3):146-9.

34. Iamarino APM, Juliano $Y$, Rosa OM, Novo NF, Favaro $\mathrm{ML}$, Ribeiro Júnior MAF. Fatores de risco associados às complicações de apendicite aguda. Rev Col Bras Cir. 2017;44(6):560-6.

Recebido em: 24/04/2018

Aceito para publicação em: 28/06/2018

Conflito de interesse: nenhum.

Fonte de financiamento: nenhuma.

\section{Endereço para correspondência:}

Vanessa Baschirotto Alexandre

E-mail: nessaab2@gmail.com vanessa.baschirotto.alexandre@gmail.com 